\title{
CPTAC Environmental/Occupational Exposure Codelist
}

National Cancer Institute

\section{Source}

National Cancer Institute. CPTAC Environmental/Occupational Exposure Codelist. NCI Thesaurus. Code C157075.

Terminology used to support the collection of patient environmental and occupational hazardous exposure data by CPTAC. 